\title{
Anomalous Phase Separation Kinetics Observed in a Micelle System
}

J. P. Wilcoxon and J.E. Martin

Organization 1153, Sandia National Laboratories, Albuquerque, NM 87185

\section{Abstract}

We report a real-time, two-dimensional light scattering study of the evolution of structure of a two component nonionic micelle system undergoing phase separation. The micelles act like molecule slug-a-beds whose domain growth is lethargic (i.e. slower than the cube root of time prediction for simple binary fluids). In fact, the growth kinetics can be empirically described as a stretched exponential approach to a pinned domain size. Although the kinetics are not yet -understood, this anomalous behavior may be due to the ability of the spherical micelles to reorganize into more complex structures.

\section{Introduction}

Micelles are surfactant aggregates with a droplet-like structure that can form in either polar or nonpolar solvents. In nonpolar solvents such as oils the hydrophobic (lyophilic) surfactant tails are exposed to form reverse or inverse micelles. Micelles formed of nonionic surfactants often phase separate when the temperature is increased or decreased (inverse systems). The critical point behavior of these systems has been the subject of several investigations[1-5] and it has been established[2-5] that they fall into the same universality class as simple binary fluids, although the critical concentration is quite low due to the large size of the micelles compared to the solvent. In particular, static properties, such as the divergence of the correlation length and the osmotic compressibility, can be expressed as power-laws in the reduced temperature with Ising exponents.

However, the universal Ising-type critical behavior of these systems does not extend deeply into the two phase region. Several years ago we observed [6] that a sample equilibrated in the two-phase region exhibits unusually strong scattering due to structures of enormous size. This novel scattering occurs in both inverse and normal micelles. It is reasonable to expect the formation of large structures to affect the kinetics of phase separation. In this paper we report the first study of the evolution of structure in a micelle system, in an attempt to understand the origin of these structures.

\section{Results and Discussion}

To study the kinetics of phase separation requires the ability to determine structure as a function of time. Traditional one-dimensional light scattering instruments must repetitively scan through a sequence of angles, with the result that data are acquired in an interval during which the structure is evolving, so that the temporal resolution is compromised. In these experiments we used a two-dimensional, time-resolved light scattering instrument that is based on currently available video and computer technology.

Radially-averaged scattering data taken during a $9 \mathrm{mK}$ quench are shown in Fig. 1 (although our time resolution is $33 \mathrm{~ms}$ we have only shown a few data sets for clarity). An unstable concentration fluctuation that intensifies with time and moves toward $\mathrm{q}=0$ is clearly observed. The momentum transfer $\mathrm{q}$ is just $4 \pi n \sin \theta / \lambda$, where $\mathrm{n}$ is the refractive index, $\theta$ the scattering angle and $\lambda$ the wavelength. A significant feature of the data is the pronounced maximum, or spinodal ring, that occurs at a scattering wavevector $\mathrm{qmax}$ and has an intensity Imax. The peak position and amplitude have simple physical interpretations: the characteristic domain size is just $\mathrm{L}(\mathrm{t})=2 \pi / \mathrm{q} \max$ and the characteristic domain mass- i.e. the domain mass within a domain size, is just proportional to $I_{\max }$. Key issues are the structure of the domains and their growth kinetics.

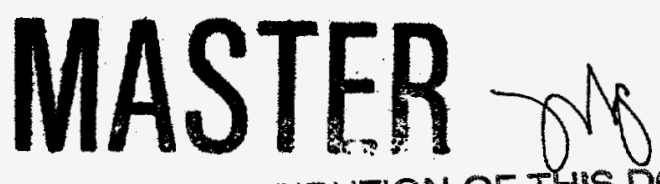

DISTRIBUTION OF THIS DOCUMENT IS UNLIMITED 


\section{DISCLAIMER}

Portions of this document may be illegible in electronic image products. Images are produced from the best available original document. 
A salient feature of these data is that as soon as a ring can be resolved its peak position is already moving toward $\mathrm{q}=0$, in contradiction to the linear Cahn-Hilliard theory, which predicts exponential growth of a stationary ring at early times. In real space, the Cahn-Hilliard description corresponds to phase separation into domains of fixed size but of increasing concentration difference. Despite our best efforts to find this linear regime we were unsuccessful. Of course, one can always postulate that the linear regime occurs on time scales shorter than can be resolved, so although we can't rule out Cahn-Hilliard, we can't measure it either.

Having resolved that the domains grow from the earliest observable times, an obvious issue is whether the domain structures grow by simple enlargement, while their morphology remains statistically constant. If this is case then the scattering data will collapse onto a master curve when plotted on the universal axes $\mathrm{I} / \mathrm{I}_{\max }$ versus $\mathrm{q} / \mathrm{q}_{\max }$. In fact, a good data collapse, shown in Fig. 2, is obtained, indicating that the expected scaling of the structure does occur, at least for shallow quenches. However, the form of the collapsed data differs in some regards from standard results, as we shall now discuss.

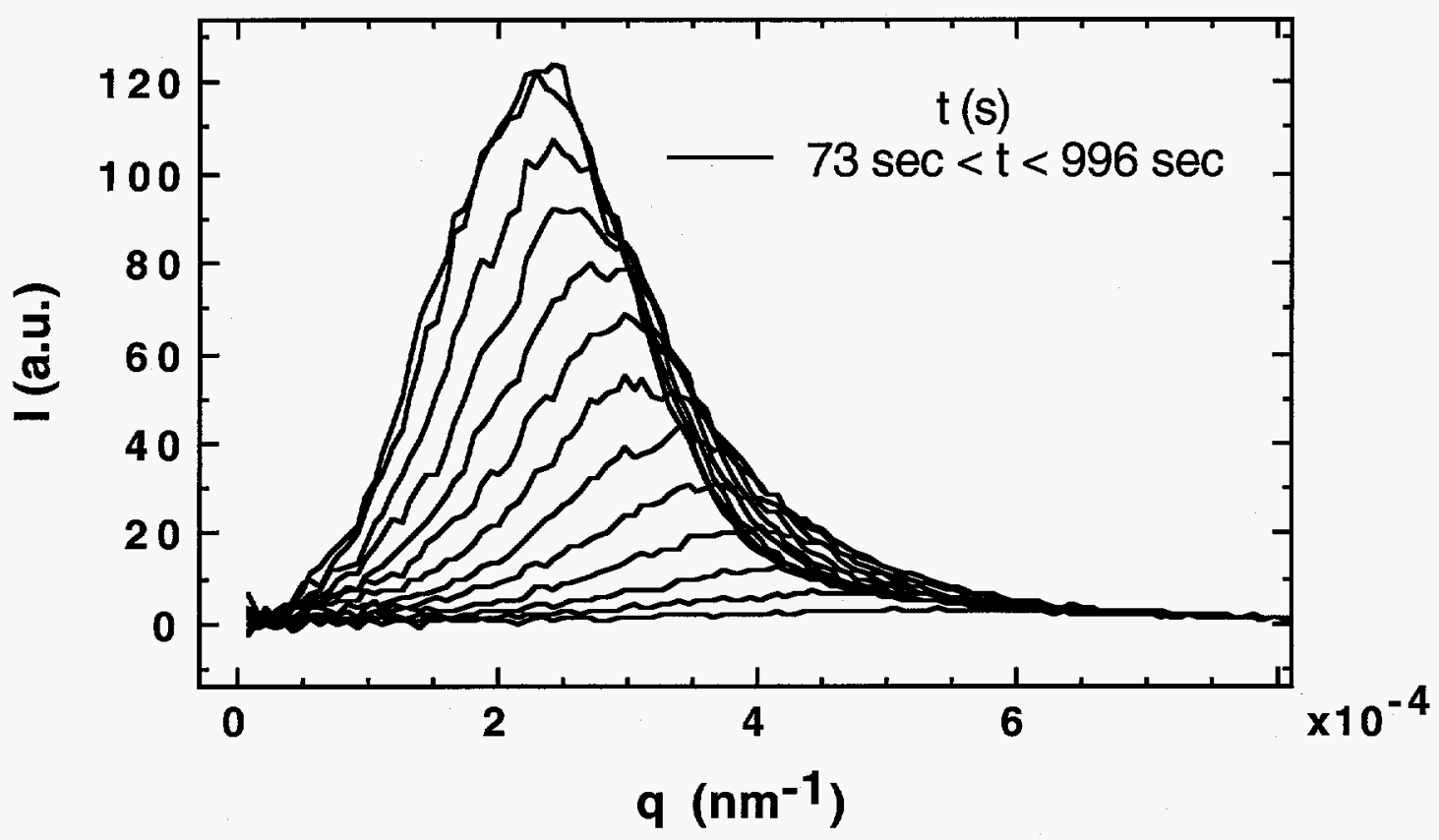

Figure 1. The growth of the radial-averaged intensity with time, as a function of the momentum transfer $q$, for a critical quench of $9 \mathrm{mK}$.

For spinodal decomposition in simple liquids (as well as other systems) Furukawa [7] has presented heuristic arguments that the scattered intensity $I(q, t)$ has the universal scaling form:

$$
I(q, t) \sim q_{\max }^{-3} f(x)
$$

where $x=q / q_{\max }$ and $q_{\max }$ is a function of time. The scaling function depends on whether the quench is along the critical isochore and has the form

$$
\begin{aligned}
f(x) & =\frac{4 x^{2}}{3+x^{8}} ; \quad \text { critical quench } \\
& =\frac{3 x^{2}}{2+x^{6}} ; \quad \text { off }- \text { critical quench }
\end{aligned}
$$


These functions have simple limiting behaviors at small and large wavevectors,

$$
\begin{array}{rlrl}
f(x) & \sim x^{2} & x<<1 & \\
& \sim x^{-6} & x>>1 ; & \text { critical quench } \\
& \sim x^{-4} & x>>1 ; & \text { off }- \text { critical quench }
\end{array}
$$

that can easily be discerned in the data. At small $\mathrm{q}$ the structure factor increases as $\mathrm{q}^{2}$, which is simply the result of having a conservation law. For large $q$ there is a $\mathrm{q}^{-4}$ decrease for an offcritical quench, which is simply Porod's scattering law for sharp interfaces. The $\mathrm{q}^{-6}$ fall-off is for critical quenches and is apparently due to a more complex interface.

The data of figure 2 do increase as $\mathrm{q}^{2}$ for $\mathrm{q}<\mathrm{q}_{\max }$ and fall-off as $\mathrm{q}^{-6}$ for $\mathrm{q}>\mathrm{q}_{\max }$, in agreement with Eq. 3. However, at higher values the $\mathrm{q}^{-6}$ fall-off crosses over to $\mathrm{q}^{-2}$, possibly indicating the formation of more complex structures, such as micellar aggregates. Alternatively, this cross-over may be due to the fact that a system near the critical point has a substantial correlation length beneath which Ornstein-Zernike scattering can be observed. In other words, critical opalescence occurs within the domain structures once the domain size is greater than the correlation length.

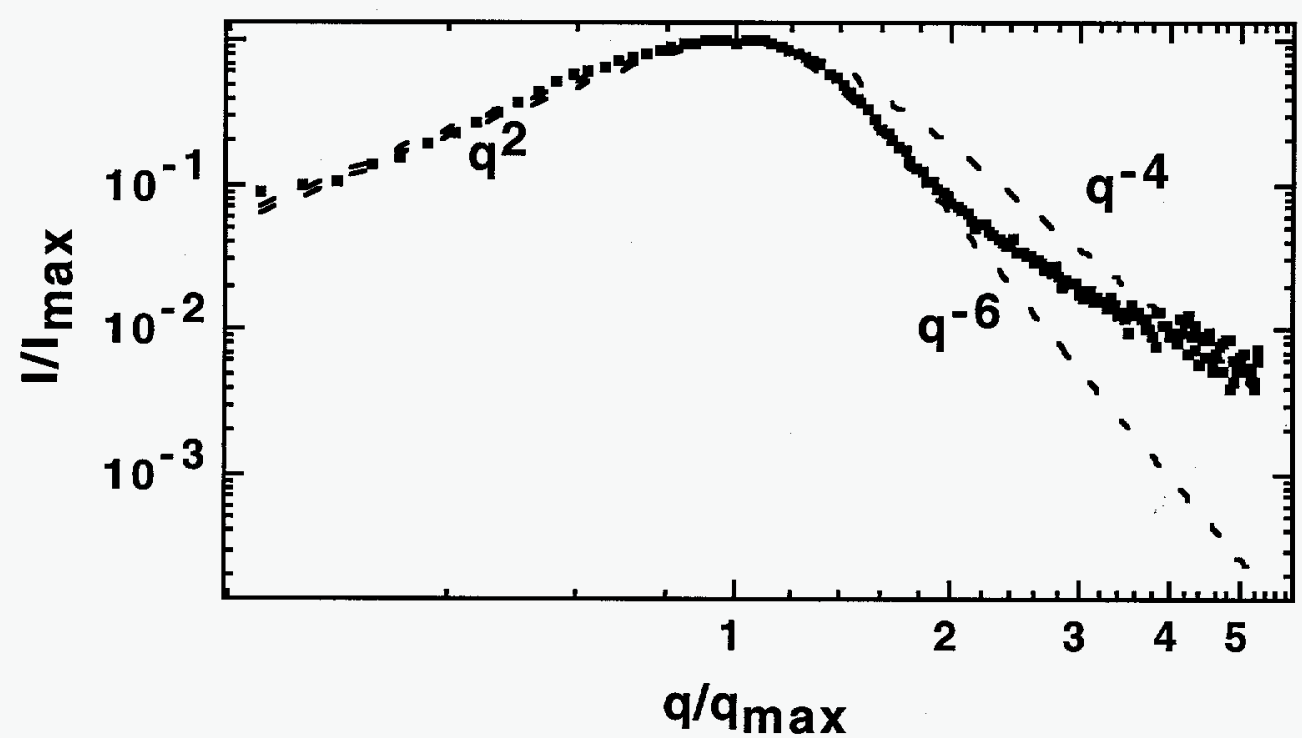

Figure 2. When the normalized intensity $I / I_{\max }$ is plotted against the normalized momentum transfer q/qmax for the $9 \mathrm{mK}$ quench a master curve results that is well described by the Furukawa [10] function for a critical quench. However, at large wavevectors a $q^{-2}$ tail is observed that is not predicted. This tail may be due to critical opalecscence within the phase separated domains.

The next issue is how the domain size depends on the domain mass. For non-mass fractal objects in three dimensions it is expected that the mass increases as the cube of a length. Since the peak intensity is a domain mass and the inverse of the peak position is the domain size we expect to observe, $I_{\max }(q) \sim q_{\max }{ }^{-3}$. Observation of this relation would imply that the coarsening of non-mass fractal domains is taking place during the phase separation process, independent of quench depth.

Scattering data for all quench experiments with $2 \mathrm{mK}<\Delta \mathrm{T}<38 \mathrm{mK}$ were quantitatively similar to the shallow quench data, albeit a crossover from critical quench to off-critical quench 
behavior was seen at large times (eq. 3), and this will be discussed later. In particular, a cubic dependence of the domain size on the domain mass was observed, and the structure factor was found to scale on dimensionless axes, with $\mathrm{q}^{2}, \mathrm{q}^{-6}$ and $\mathrm{q}^{-2}$ regimes. Evidently, the integrated measure of structure (eq. 4) is much less sensitive to morphology than the structure factor itself. However, as we shall now discuss, the kinetic data show large differences between the shallow and deeper quenches.

Following a suggestion of S. Glotzer, who has simulated spinodally decomposing systems that exhibit pinning, we then attempted to analyze our data in terms of an asymptotic approach to some pinning length, $\mathrm{L}(\infty)$, i.e.

$$
L(t)=L(\infty)\left(1-e^{-\left(\frac{t}{\tau}\right)^{b}}\right)
$$

As in the case of both power law and logarithmic fits, this function has 3 adjustable parameters, $\mathrm{L}(\infty), \tau$, and $\mathrm{b}$. Analysis demonstrated that the optimal stretched exponential exponent $\mathrm{b}$ was very close to 0.5 for all quench depths, while the pinning length $\mathrm{L}(\infty)$ was nearly constant at a value of $\sim 25 \mathrm{~mm}$. We thus chose to fix these parameters and let only the characteristic time $\tau$ vary with quench depth. These fits to eq. 4 follow the experimental data of Fig. 3 and demonstrate that eq. 4 is a good description of all our data. An important point, however, is that a functional form that approaches an asymptote can be a misleading fit unless the pinning length is reasonably close to the largest length scale in the experimental data. Our data approaches $80 \%$ of the pinning length, so we do not think that the pinning interpretation can be rejected out of hand.

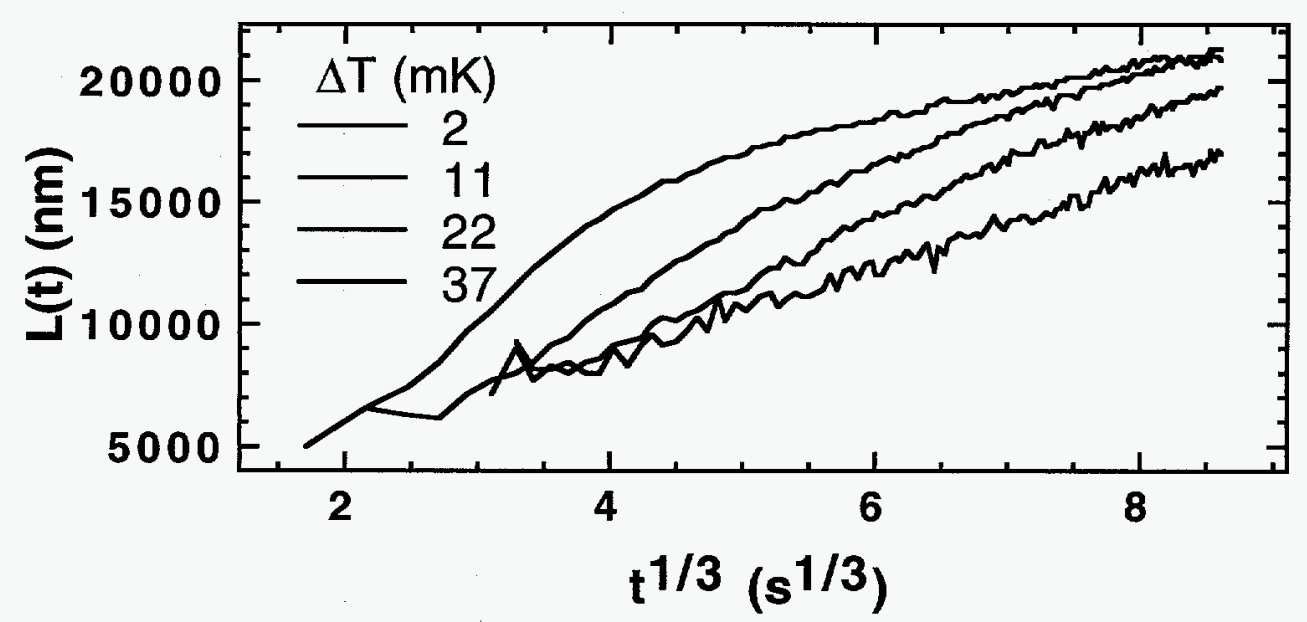

Figure 3 . The growth of the domain size as a function of time tfor four quench depths is plotted against $t^{1 / 3}$ to show deviations from Lifshitz-Slyozov growth. Significant deviations in the form of an apparent slowing down occur at large times for deep quenches. All our data are described by the stretched exponential form of eq. 4 .

The stretched exponential analysis doesn't resolve the question of whether the postulated pinning is extrinsic- due perhaps to impurities- or intrinsic, due to the ability of the spherical micelles to restructure into more complex morphologies. We do not expect impurities of a chemical nature in our samples, and this assumption is fortified by previous HPLC analysis of the surfactant used. Furthermore, any impurities would probably be quickly encapsulated by surfactants and thus rendered benign in their ability to moderate interactions between micelles. We suspect that the flattened portion of the phase diagram near the critical point leads to phases with large concentration differences even for relatively small quenches. 


\title{
Acknowledgment
}

This work was performed at Sandia National Laboratories, Albuquerque, NM and supported by the U.S. Department of Energy under Contract No. DE-04-76DP00789.

\section{References}

1. M. Corti and V. Degiorgio, Phys. Rev. Lett. 55, 2005, (1985).

2. J.P. Wilcoxon and E.W. Kaler, J. Chem. Phys. 86, 4684, (1987).

3. G. Dietler and D.S. Cannell, Phys. Rev. Lett. 60, 1852, (1988).

4. K. Hamano, N. Kuwahara, I. Mitsushima, K. Kubota, and T. Kamura, J. Chem. Phys., 94, 2172, (1991).

5. K. Hamano, N. Kuwahara, I. Mitsushima, K. Kubota, and J. Ito, Phys. Lett. A 150, 405, (1990).

6. J.P. Wilcoxon, J. Phys. Chem. 94, 7588, (1990).

7. H. Furukawa, Physica, 123A, 497, (1984).

\section{DISCLAIMER}

\begin{abstract}
This report was prepared as an account of work sponsored by an agency of the United States Government. Neither the United States Government nor any agency thereof, nor any of their employees, makes any warranty, express or implied, or assumes any legal liability or responsibility for the accuracy, completeness, or usefulness of any information, apparatus, product, or process disclosed, or represents that its use would not infringe privately owned rights. Reference herein to any specific commercial product, process, or service by trade name, trademark, manufacturer, or otherwise does not necessarily constitute or imply its endorsement, recommendation, or favoring by the United States Government or any agency thereof. The views and opinions of authors expressed herein do not necessarily state or reflect those of the United States Government or any agency thereof.
\end{abstract}

